\title{
Urgences
}

\section{L'image espionne}

\section{Paul Bleton et Chritian-Marie Pons}

Numéro 32, mai 1991

Lectures de bandes dessinées

URI : https://id.erudit.org/iderudit/025648ar

DOI : https://doi.org/10.7202/025648ar

Aller au sommaire du numéro

Éditeur(s)

Urgences

ISSN

0226-9554 (imprimé)

1927-3924 (numérique)

Découvrir la revue

Citer cet article

Bleton, P. \& Pons, C.-M. (1991). L'image espionne. Urgences, (32), 59-72.

https://doi.org/10.7202/025648ar d'utilisation que vous pouvez consulter en ligne.

https://apropos.erudit.org/fr/usagers/politique-dutilisation/ 


\section{L'image espionne \\ Paul Bleton et Christian-Marie Pons}

Le tableau n'est pas d'abord objet de connaissance, support et provocation d'une conceptualisation. Il est être producteur de plaisir.

Louis Marin, « La description de l'image à propos d'un tableau de Poussin ».

À propos d'une BD policière, nous avions déjà étudié la part du lecteur dans la mise en œuvre du sens et souligné combien la coopération interprétative définit la lecture à la fois comme déchiffrage et comme gamberge: depuis la lecture fidèle, jusqu'à la perplexité inventive devant les signes obtus, en passant par l'invitation à la détection et à l'abduction prévisible dans un tel genre ${ }^{1}$. En nous plaçant cette fois-ci non plus en aval mais en amont de la sémiosis, nous aimerions ici, par le commentaire d'un fragment de La porte d'Orient de Giardino ${ }^{2}$, décomplexifier un dispositif visuel singulier, triplement stratifié: scène phénoménologique préformant tout un genre paralittéraire, figuration d'un motif narratif convenu et acte de lecture complexe (figure 1, page suivante).

Décidément, l'encyclopédie du lecteur qui lui permet d'en savoir assez sur l'univers du spionspiel et ses doubles fonds aura naturalisé ce retournement: Magda a sans doute, par sa séduction, dissimulé son rôle réel à Fridman. C'est que la fréquentation du récit d'espionnage enseigne une cruelle leçon: les apparences sont trompeuses; et la littérature de tous les temps, cette autre: que celles de la femme peuvent même être fatales. La séduction comme arme du combat de

1 120, rue de la Gare de Tardi, d'après Léo Malet. Voir Paul Bleton et Christian-Marie Pons, "Mégots et journaux: signes interprétables et obtus ", dans Mieux vaut Tardi, sous la direction de Jacques Samson, Montréal, Analogon, 1989.

2 Vittorio Giardino, La porte d'Orient, coll. "Les aventures de Max Fridman ", $n^{\circ} 2$, Grenoble, Glénat, 1986. 


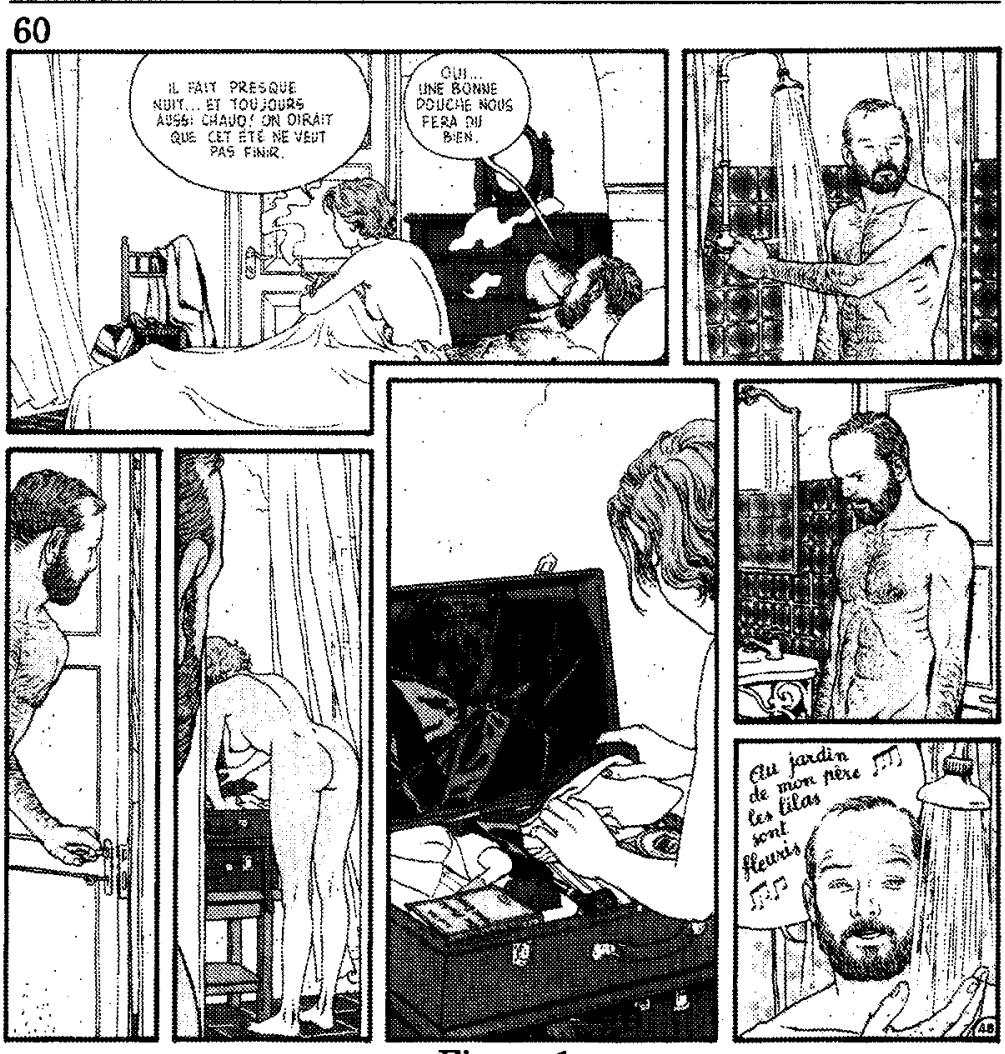

Figure 1

l'ombre et la nudité, censée vêtir la Vérité sortant de son puits, comme paradoxale apparence... Fridman, en bon sceptique, en sait au moins autant que le lecteur à ce chapitre, lui qui accorde plus de poids à ce qu'elle fait qu'à ce qu'elle dit, et à ce qu'elle fait cachée au détriment de ce qu'elle fait avec lui. À vrai voir, la vérité de Magda nue serait qu'elle est une trompeuse. Une trompeuse? certainement: n'allez surtout pas croire que son geste puisse être interprété innocemment, comme quelque charmant défaut de son sexe, quelque anodine curiosité; dans l'univers du spionspiel, la curiosité est un acte de métier, la curieuse certainement une espionne, et l'espionne une ennemie. Au commencement était le retournement; à l'origine de l'image, la nudité est duplice, et le spectacle du corps nu n'est plus immédiat, il est interprété - et doublement - par au moins deux voyeurs 
méfiants, par deux regards dissimulés redoublant la scène: enchâssement des regards qui voient sans être vus, Magda, Fridman, lecteur enfin...

Telle est bien la scène phénoménologique de base de tout l'univers de l'espionnage, scène modélisante du regard de combat, mais pas des yeux qui s'affrontent: du regard dissimulé, de l'observation clandestine

"X épie Y"

Comme la simplicité de sa structure rend cette scène indéfiniment disponible, la complexité s'obtiendra par simple réitération,

"Z épie " $X$ épie $Y$ ", etc.

voire "Y épie " $\mathrm{X}$ épie $\mathrm{Y}$ " "...

Et tel est bien son sens - ni curieuse, ni voyeur, l'un et l'autre espions. L'indécision ne peut durer trop longtemps; pour survivre, chacun doit "placer' rapidement son partenaire de jeu; la sapience spionspiel qu'auteur, lecteur et personnages partagent, cette sapience le dit bien: l'amour, c'est la guerre poursuivie par d'autres moyens.

Pour le lecteur, le cas de Fridman est plus clair encore. Peut-être à son corps défendant, il n'en est pas moins un agent; pas mercenaire, mais professionnel retiré des affaires, avec une longue expérience allusivement signalée, un vrai passé bédéistique (second volume des Aventures de Max Fridman, l'une des rares séries BD consacrées au seul espionnage) et une famille spirituelle, celle des héros malgré eux, des espions contraints, fragiles mais victorieux, agents déterritorialisés d'Eric Ambler et de Graham Greene. Ainsi, même très loin de l'érythique modèle des espions de papier professionnels et mercenaires comme les 007 et autres sas, Fridman n'est pas inouï; depuis les années trente, de l' espion contraint, fragile mais victorieux, le genre a fait un "emploi", comme on dit au théâtre; ses compétences, en particulier celle consistant à ne pas se laisser piéger, sont prévisibles dans une histoire d'espionnage - condition sine qua non de la survie en milieu hostile. Et que la beauté de Magda soit plus touchante qu'explosive ne change rien à l'affaire; sortie du puits, sa nudité l'a révélée trompeuse. De ces deux-là, les actions sont claires, en regard des règles de l'univers de 
62

référence et des lois du genres - à défaut que leurs motivations respectives le soient également.

Mais peut-être le lecteur est-il plus suspicieux encore. Certes, comme Fridman il accepte la conclusion implicite qui «place» la partenaire du jeu amoureux comme concurrente dans le jeu de l'espionnage. Mais il se sent bien voir Fridman: il peut avoir de la sympathie pour lui, l'«identification" au héros ne va quand même pas jusqu'à quelque psychotique symbiose: le lecteur voit Fridman épier, puis il le voit sous la douche. Vision spontanée? Amateur d'espionnage ou bédéphile, il n'est pas si naïf pour y croire au-delà de cette première lecture où il se contente de dévorer l'histoire, où la vitesse, le désir du dénouement sont ses seuls plaisirs. Alors, qui donc lui refile de telles informations? Quelle caméra invisible entre dans l'intimité amoureuse et professionnelle des protagonistes? On parle d'un certain Giardino, sans prénom ce qui est déjà un peu louche. Il faut enquêter ailleurs, procéder par recoupements pour lui trouver ce prénom manquant ${ }^{3}$; et s'étonner de ce que l'information sur ce héros juif de patronyme, français de passeport mais cosmopolite de mode de vie nous vienne d'un Italien (confirmé par le copyright qui donne Milano Libri ${ }^{4}$ comme origine de l'information et par d'autres recoupements établissant nettement l'existence d'une filière franco-italienne Corto Maltese-Vécu)... Attention, ce Giardino n'est pas un sage qui s'occuperait de son jardin et de ses seuls oignons; l'espionnage, on l'a vu, est spontanément international et relève plutôt d'une déterritorialisation, à partir d'un territoire peut-être moins national qu'esthétique; l'Italie renaissante n'a-t-elle pas inventé l'espionnage moderne et les maîtres-espions de la BD contemporaine ne se nommentils pas Giardino, Pratt, Micheluzzi ${ }^{5}$ ?... On ne saurait rester indifférent à deux observations contenues dans le dossier consacré à notre informateur: sa ressemblance criante avec Fridman (figure 2) et cet aveu de nomination croisée et refoulée: «J'avais aussi envisagé [le nom] de Liebman, mais c'est le nom de jeune fille de ma belle-mère et elle aurait pu y

3 Comme dans le dossier "Vittorio Giardino" que lui consacraient Les cahiers de la bande dessinée, $n^{\circ} 71$, sept.-oct. 1986.

$4 \quad$ Les cahiers de la bande dessinée, $n^{\circ} 71, p .36$.

5 Paul Bleton, "La taupe fait des bulles, bande dessinée et espionnage", à paraitre. 


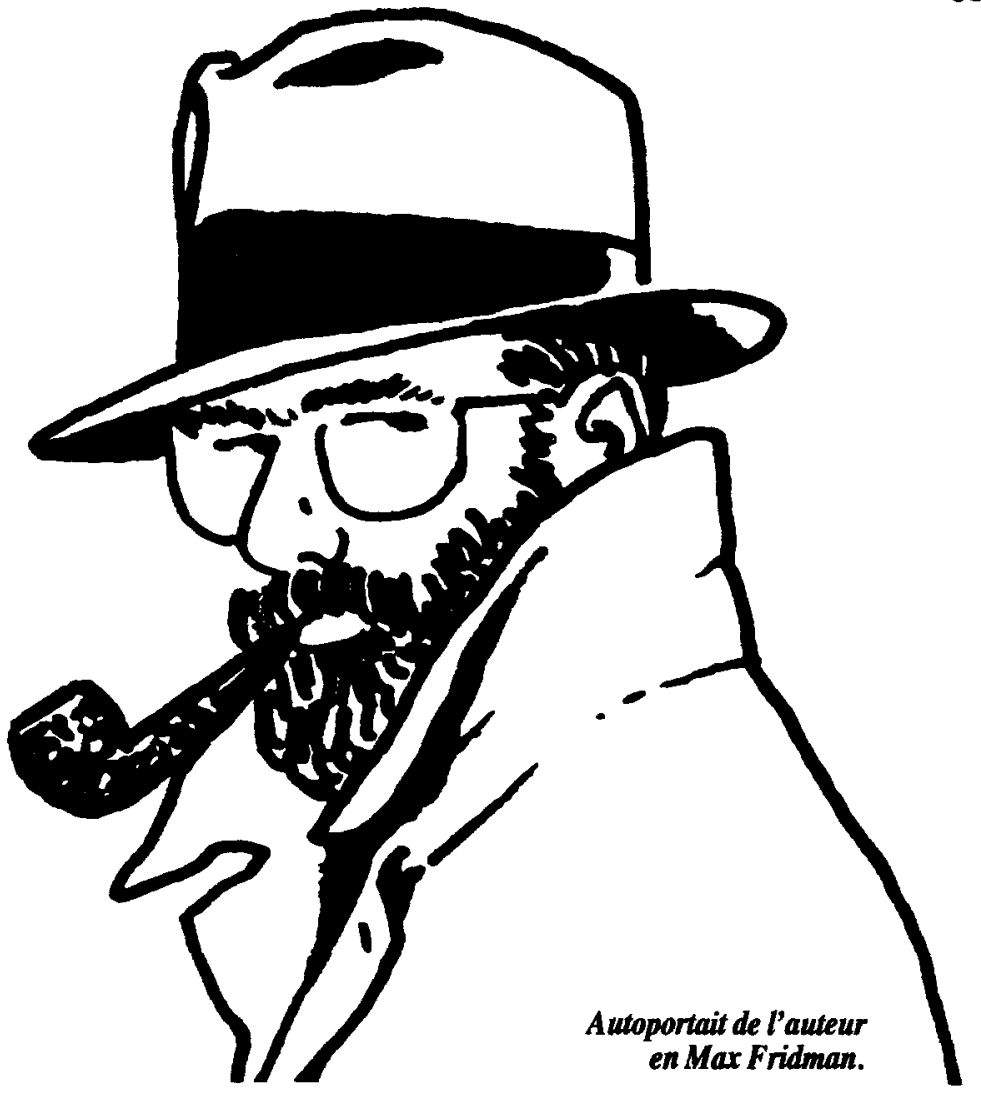

Figure 2

voir je ne sais quelle allusion ... (rires)" 6 . L'information que nous lui devons, une série d'icônes, présuppose une effraction; la scène se déroule en effet dans la salle de bain, mais si effraction il y eut, ce ne devait pas être celle de l'intimité du lieu mais plutôt celle de l'intimité d'un regard et de ses avatars: regard perdu et geste machinal du chasseur qui entend un bruit suspect, regard professionnel, focalisé mais invisible, attentif et clandestin de l'agent surprenant une collègue espionnant, regard perdu de la gamberge et de la déception, regard frontal

6 Thierry Groensteen, « Entretien avec Vittorio Giardino ", Les cahiers de la bande dessinée, n॰ 71 . 
64

enfin, faussement jubilatoire. Regard frontal qui vraisemblablement accroche cet autre regard que la thématisation désigne en creux: celui de notre informateur ou de son objectif planqué. Comment Giardino a-t-il pu préparer ce dispositif d'observation clandestine alors que le séjour des deux protagonistes dans cette chambre n'était ni prémédité ni prévisible? Quel crédit peut-on faire à un informateur devenu si proche de son objectif assigné ("objectif» dans son sens militaire, pas optique, "proche" dans son sens psychologique, pas topologique); information, certes; mais information visiblement mise en scène. Et en fait, pour qui donc travaille ce Giardino?

Et si, suspicieux, le lecteur en vient à percevoir que l'informateur le manipule, met en scène (dans quel but?) l'information, c'est bien qu'il est lui aussi partie prenante, assigné à quelque mission en terrain hostile, à quelque enquête rendue difficile par la surabondance d'indices, par les leurres que le récit, la figuration ou les personnages lui tendent... En fait, le lecteur, qui lui ne signe pas des albums de $B D$, qui n'est pas interviewé par des revues spécialisées, le lecteur n'en voit pas moins la même chose que Giardino c'est même là sa dépendance - alors que sa lecture lui démontre constamment et à sa confusion qu'il en sait moins que Giardino ! Ce dernier a en effet accès au passé et au futur de l'histoire qu'il rapporte, il peut voir ce qui se passe hors champ et entre les vignettes, alors que le lecteur est dans l'immédiateté du présent présenté, encadré, rétréci.

Magda, en espionne supposée; Fridman, en espion patenté; Giardino, en informateur retors; le lecteur, en enquêteur manipulé... il se dégage de cette artificieuse documentation à partir de quoi doit se forger une opinion, une impression ou un sentiment, il se dégage une idée paradoxale: l'image elle-même n'aurait-elle pas son propre agenda? ne serait-elle pas la plus machiavélique? cinquième mousquetaire de l'ombre, cinquième colonne?

Reprenons. à la généralité du noyau phénoménologique modélisant le genre,

"X épie Y"

la séquence [48:6/7/8] ajoute un déplacement du point de vue, un investissement de la profondeur: travelling avant, courbe comme il se doit dans une telle histoire: 
[48: 6]: approche clandestine de l'épieur fourbe, qui avance de biais, et qui par le jeu de la porte entrouverte, souligne la profondeur, la différence entre deux plans (la salle de bain/la chambre);

[48: 7]: ambiguïté de cette scène dont la double valeur est immédiate (espionnage/érotisme) à cause des fesses nues sur une diagonale invisible mais fortement organisatrice (regard de Fridman) et du sein sur une seconde qui poursuit le virage sur la gauche et offre en contrepoint à l'évidence des fesses nues le plus discret jeu de mains de Magda qui fouille;

[48: 8]: plus discret mais plus déterminant, puisque l'ambiguité introduite par le regard de Fridman (espionnage, érotisme?) est maintenant hors champ, la nudité même de Magda s'estompe, c'est l'espionnage de Magda qui compte, c'est la diagonale invisible de son regard qui organise la fin du virage à gauche. Ici, blocage du travelling avant qui bute sur le couvercle ouvert de la valise ${ }^{7}$ thématisant à plat le point de fuite (les courroies qui maintiennent à plat le costume et qui se croisent à la place du cœur).

Il semble bien ici, d'un côté que les espions représentés ne soient pas les seuls à épier, que d'autres yeux que les leurs soient sur la même affaire; et d'un autre côté que les bons vieux services secrets français, russes ou autres d'avantguerre soient à leur tour doublés par une organisation d'un tout autre ordre, vraisemblablement ni naïve, ni innocente, qui ne laisse pas sa place en fait de leurre et d'intox, de simulacre ${ }^{8}$.

Ainsi, du côté du "regard espiant»: un visage représenté de profil permet au lecteur de l'observer sans être vu par lui, espionné donc (c'est celui de Fridman entrouvrant la porte de la chambre); un visage représenté de $3 / 4$ arrière permet d'observer, toujours sans être vu, ce qui déjà est espionné par

7 Formant une « surface consolidation", selon le terme de Erwin Panovski pour évoquer la présence dans la représentation d'une surface solide et opaque arrêtant l'ceil dans sa fuite vers l'horizon et pour cantonner celui-ci dans une sorte de profondeur plate ("a slab of volume"). A propos de BD, Alain Rey parle aussi de "trouée de la page ou d'écrasement au milieu plat de l'écriture". (Alain Rey, Les spectres de la bande, coll. «Critique", Paris, Minuit, 1978).

8 Christian-Marie Pons, "L'image sorcière", dans Littérature pour la jeunesse, Les cahiers de l'APFUCC, II, 2, Victoria (BC), 1988. 
66

l'espion qu'on espionne (c'est Fridman encore, l'image suivante, et l'objet de son regard: les fesses et gestes de Magda fouillant la valise); le regard oblique d'un visage représenté de face, c'est enfin le regard de qui se sait observé mais ne veut, en le fixant de face, dévoiler au lecteur espion qu'il en connaît la présence (par ruse).

Bien sûr, entre observateur et observé (l'observé, comme finalité voulue du regard, peut être un acteur ou l'action de celui-ci), entre le su et l'insu, il y a de multiples figures possibles, dont celle, importante quand il est question de regard, du paraître (le faire versus le faire-semblant: c'est toute la rhétorique de la ruse: tromperie, leurre, intox, secret, simuler pour dissimuler, double jeu, etc).

Si l'on se cache pour voir, c'est que l'autre se cache pour faire: voir sans se faire voir, ou faire sans se faire voir. Le secret, l'insu est souvent des deux cotés du regard (observateur et observé). L'insu absolu (l'ignorance) est rare et ennuyeux, puisqu'il annule purement et simplement la réalité des choses, autant dire qu'il n'existe pas; l'insu partiel, en revanche, c'est l'opacité du secret, du mystère (je sais qu'il y a quelque chose, mais je ne sais pas quoi; je sais qu'il y a quelque chose, mais je ne sais pas si c'est cela; je sais que je ne sais pas...), le secret comme mélange de su et d'insu.

Ce mélange peut être l'effet d'une intention (créer un mystère de toute pièce: le leurre du Glaros dans Rhapsodie hongroise, ou la notion même de suspens narratif en général, ou encore la pudeur comme stratégie érotique), comme il peut être la conséquence (involontaire) d'une impureté du su et/ou de l'insu: vouloir cacher sans y parvenir totalement (le secret), mais aussi vouloir montrer sans y parvenir complètement (les limites de la représentation).

Le regard aux aguets qui fonde l'espionnage suppose une profondeur, une valise dissimulant quelque secret; il a le besoin de quitter la surface, de dépasser le regard qui effleure; énergique, il rebondit sur la * surface consolidée * et son point de fuite - ici mal nommé - pour s'enfoncer dans les chemises qui certainement planquent un dossier ${ }^{9}$. Un dossier ou

9 On l'aura compris, cette valise à double fond s'ouvre sur * The case for case reopened " de Fillmore (1977), et sur l'usage qu'une sémiotique de la BD 
au moins un message secret, comme le titre d'un livre dans une langue étrangère, sens qui s'exhibe et se dérobe à la lecture, Die Flucht ohne Ende (1936), « fuite sans fin * de ce Roth, paronyme de la mère de Fridman, Ruth [44: 2], secret œdipien? synonyme d'un ex-militant catalan républicain, secret politique ${ }^{10}$ ? Ou un message révélant que le spionspiel n'est pas seulement affaire de secret, mais aussi de tromperie, de pipes - le mystère de la pipe jaune, pipe magdalénienne présente dans les strates archéologiques de l'histoire commune de Fridman et de Magda, fumée ailleurs [6:6] au son de * a fine romance with no kisses", gonflée comme un sax "when a lovely flame dies smoke gets in your eyes" [27: 2], bourrée en [11: 5] de perplexité " cette femme me rappelle quelqu'un...", mystère éclairci par le lecteur: cette pipe usagée, ne salissant même pas la chemise immaculée sur laquelle elle repose, rappelle quelqu'un...: ceci n'est pas une pipe, tout juste une fumisterie. Dans la profondeur de la valise, le rappel que Fridman est officiellement négociant en tabac: écran de fumée et homme de paille, encore une pipe de contée $^{11}$. Vraie pipe ou fausse piste.

\section{Quand, oblique, le regard transfuge.}

Reprenons encore. Voilà que tombe sous nos yeux la trace d'une banale histoire d'espionnage: elle, en ingénue voyageuse, sait attendrir le vieux pro aux allures rangées comme ses pipes. $\mathrm{Au}$ prix d'un peu de son corps, elle gagne l'la possibilité de jeter un œil au creux des effets personnels du monsieur qui y cacherait l'information qu'elle cherche. Quant à lui, n'ayant pas cédé tout son esprit aux avances de la dame, il nous montre en bon espion qu'il lui reste plus d'un tour dans son sac. Occasion d'une scène réjouissante, fidèle au genre: l'espionne espionnée,

peut obliquement faire de cette problématique de linguiste (case réouverte?). Voir C. Fillmore, "The case for case reopened", dans Syntax and Semantics, 8, Grammatical Relations, sous la direction de P. Cole et J. M. Sadock, New York, Academic Press, 1977.

10 Voir Vittorio Giardino, Rhapsodie hongroise, coll. “ Les aventures de Max Fridman ", Grenoble, Glénat, 1982.

11 Quant à la confession de Slatek dans la fumée du narguilé, comment Fridman, spécialiste en tromperie pourrait-il y croire [51]? Contre-épreuve: pensez-vous qu'avec un nom comme Bonnefoi, un agent ait quelque chance de finir vivant de telles histoires? Voir Rhapsodie hongroise. 
68

avec un zeste de peau. L'histoire pourrait en rester là et passer à l'épisode suivant. L'aventure de l'espion et de l'espionne au Pavillon Turba pourrait passer, comme on dit, inaperçue.

Mais il y a ce détail, sans doute le rapprochement de cette porte qu'il entrebâille pour l'observer à son insu et cette autre fente, blanche celle-ci, entre l'image et la suivante; ou encore ce constat que lui qui observe Magda fouillant est lui-même représenté sous le même angle ( $3 / 4$ arrière) que celle qu'il espionne et qu'à son tour, donc, lui aussi est observé à son insu pendant qu'il observe l'espionne qui fouille une valise dont nous-mêmes, enfin, pouvons visiter le contenu; le souvenir transparent qu'un certain Giardino, Vittorio Giardino disent les dossiers, aurait favorisé cette entrevue; l'impression surtout à suivre le regard de l'homme de retour dans sa douche, porte refermée et n'en finissant pas de regarder la blancheur des chemises soulevées par les mains de l'espionne; ou encore ce clin d'œil, direct, de l'homme ironique, chantant à tue-tête sa comptine inspirée aux céramiques de son (double?) fond de douche, comme si non seulement les murs avaient des oreilles, mais aussi des yeux.

Car, en fait de regard représenté, il est aussi celui de face, très remarquable en ce qu'il convoque immanquablement le lecteur. Ici, le voyeur est vu voyant par ce qu'il regarde, surpris dans son intrusion (c'est le trompe-l'œil final que Fridman nous décoche en fredonnant sous sa douche).

Or, notamment dans cette scène, la présence reconnue d'un observateur ne peut qu'être dérangeante (dans un monde d'espionnage et de secret doublé ici d'intimité dénudée, les témoins sont rarement bienvenus), soit alors complice de l'observé qui nous sait l'observant (entre espions du même bord, on peut bien se montrer un peu nus, au moins jusqu'à la ceinture). Complice puisqu'il nous signale que nous avons été vu voyant (à l'inverse du regard oblique qui fait semblant de ne pas voir celui qui observe et qui, trompé, se croit toujours caché).

D'une histoire de regard et de nudité à l'autre, le phénomène est vieux, et s'il est en plein l'enjeu préféré de l'espionnage, il est aussi celui plus général de la représentation, de l'image.

Déjà, dans Rhapsodie hongroise, le doublet est clairement identifié [42-43]: Cléo-Ingrid la trompeuse, agent double et cheville du vaste leurre que poursuit Fridman (et Giardino) 
dans le premier récit, c'est aussi Olympe, tenancière d'un bordel au miroir sans tain montrant (sans être vue) l'espion d'en face, face à une croupe empruntée ${ }^{12}$ dans une activité résolument sexuelle. C'est surtout Olympia: la pose, le sofa, l'escarpin légèrement déchaussé, la servante noire, le chat (assis ici sur son double), et le regard de face (figure $3, \mathrm{RH}: 42$ ), tout est là pour évoquer le scandale célèbre de la toile de Manet (1863). De ce tableau (tout comme Le déjeuner sur l'herbe) l'analyse des historiens de l'art est connue: ce n'est pas tant la nudité de la courtisane qui choque, mais bien, par l'intermédiaire du peintre, qu'elle nous regarde la regarder nue. La pudeur consiste moins à se cacher qu’à savoir ne pas dévoiler au voyeur qu'on le sait s'adonner au voyeurisme (la pudeur est bien une ruse). Fin de parcours, cette image évidente, bien connue elle aussi, de A. Dürer, dessinateur de la femme couchée (1525) ${ }^{13}$ (figure 4). Â se rincer l'œil, qu'on ne s'y trompe pas tout à fait: l'œil du peintre est une serrure où coller le nôtre ${ }^{14}$.

Et qu'entend-on alors? Une chansonnette cousue de fil blanc. À propos du giardino, un reliquat qui n'échappe pas: la

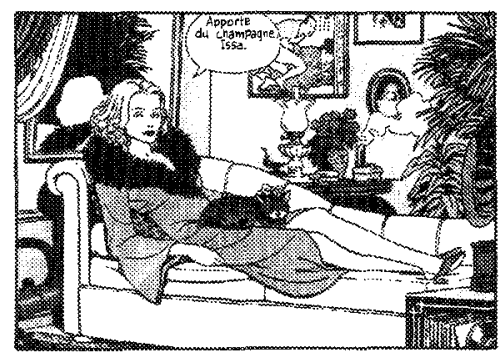

Figure 3

12 N'en cherchez pas d'autre: il n'y a bien que deux vues de derrière en tenue d'Eve dans les deux albums de la série Max Fridman: celle de Magda fouillant la valise et celle de la putain de la Lune verte.

13 « Dans le jeu des figures du tableau, un "je", à la fois anonyme et constamment présent comme absent dans la représentation " (Louis Marin, “ La description de l'image à propos d'un tableau de Poussin", dans Communications, $n^{\circ} 15$, Paris, Seuil, 1970, p. 194 ).

14 Question de point de vue, bien sûr. Par exemple, ici, dans La porte d'Orient [26], c'est aussi a travers une grille que le lecteur peut lire ce billet discret qu'une diseuse de bonne aventure vient de remettre à Magda. Nous sommes si bien placés que cette information livrée au lecteur n'est pas accessible à Fridman lui-même ( figure 5, PO, 26: 6-7). 


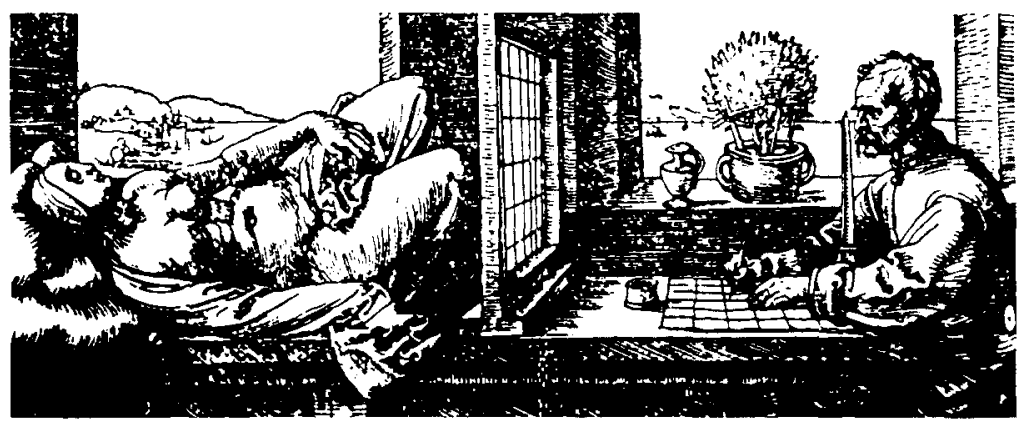

Figure 4
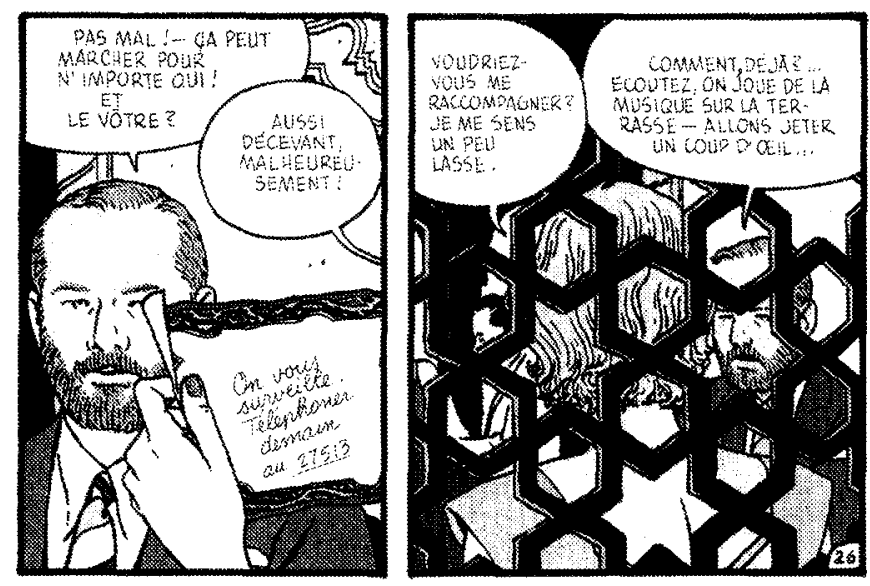

Figure 5

touchante allusion filiale du personnage à l'auteur de ses jours; mais surplus, la certitude que cet espion, rhapsode qui en a vu d'autres, s'amuse à nous voir l'écouter ou, le rusé, profite de notre proximité intra-muros pour passer l'information au nez, et à la barbe, des pages qui le renferment; inaugurant sous sa douche turque ce que quelques années plus tard la $\mathrm{BBC}$ rendit célèbre: les messages farfelus déguisant des secrets d'État. "Auprès de ma blonde, qu'il fait bon, fait bon dormir...", ça, il 
suffit d'avoir assisté au haut de la page pour en saisir l'inspiration et l'hommage; mais la suite «Que donneriez-vous belle pour avoir vot' mari ? "À quoi joue donc ce Fridman qui feignait encore ignorer sept pages plus tard les projets bien gardés de Magda, alias Mme Stern, et qui semble déjà, doublant le récit, savoir la raison (profonde) de la fouille de sa valise (retracer pour le sauver David Stern, transfuge et traqué)?

Quant à "Je donnerais Versailles, Paris et St-Denis...", c'est de la fiction, de la clairvoyance suspicieuse, ou de la prolepse éhontée: comment induire déjà en 1936, que la femme prête à tirer son savant homme des griffes du NKVD, était prête à le faire aux dépens des fragiles alliances francosoviétiques, érigées en rempart aux projets nazis dont les bottes finiront par piétiner, quatre ans plus tard, le traité de Versailles ${ }^{15}$ en occupant la capitale française et ses environs.

Oblique, le regard représenté est aussi celui de celui qu'on espionne qui écoute (espionnage acoustique: c'est Fridman bouche bée, la contradiction de son geste et de son regard nous le montre tendant l'oreille [48: 5]). La bande dessinée se laissant (au risque d'être trahie) doubler, par convention, d'une bande sonore, cette implication acoustique n'est pas vaine. Regard qui écoute [48:5] ou oreille qui voit [48:7], c'est à ne plus savoir qui de l'œil ou d'Eustache est trompé. Ce regard qui nous regarde sous la douche, et qui chante pour l'oreille des murs, ne l'oublions pas, reste de papier, de ce côtélà de la représentation; transfuge fuyant le point de fuite, il demeure sous surveillance ${ }^{16}$. Bien sûr, espion amateur de chair et d'os, le lecteur ne peut traverser, lui, le vitrage du plan de représentation, si transparent soit-il; notre intrusion par effraction dans la salle de bain n'est qu'un leurre; biaisé, c'est par réfraction que ce regard et son chant nous atteint ${ }^{17}$.

Avec son noyau phénoménologique, l'univers propre à l'espionnage colonise le système perspectif, la profondeur et le trompe-l'œil, et leurs objets respectifs — voiles de la porte

\footnotetext{
15 Celui qui mit fin, en 1919, à la Première Guerre mondiale.

16 Ultime mission d'une image espionne: faire croire au lecteur que le plan de représentation a été dérobé.

17 . Il faut se méfier des apparences", revoir la leçon de l'aquarium: la dernière bulle du poisson rouge parmi les algues ichtyophages dans Rhapsodie hongroise [36: 4].
} 
entrebâillée, de la fumée, du rideau de l'eau qui tombe, du rideau de douche, du rideau de la fenètre pour la première, miroirs dans la chambre et dans la salle de bain pour le second, miroirs qui a défaut de réfléchir ce qu'ils voient nous font penser à la nature de l'image... En retour, diagonales invisibles et architectoniques débordent la simple composition de la seule vignette pour former le découpage de la séquence de vignettes, pour organiser le micro-motif issu de " $\mathrm{X}$ épie $\mathrm{Y}$ ": diagonale partant du regard abasourdi de Fridman, passant par celui inquisiteur de Magda pour revenir sur la scène originaire des mains traîtresses et farfouilleuses, diagonale partant du mème regard, passant par le point de jonction du visage et de l'épaule de Magda pour aboutir sur sa pipe...

Il nous faudra bien, toujours suivant Fillmore, nous pencher nous aussi sur le cas de la case à la valise réouverte. Qui croyons-nous être, juste dans le dos de Magda nue (Dieu sait où traîne la main) qui ne nous entend pas, et lisant par dessus son épaule le titre d'un bouquin d'outre-Rhin à défaut d'autre découverte.

Tout comme les apparences, la profondeur leurre; la fuite sans fin, ce n'est pas seulement celle, spatiale, iterfactive, de Stern ${ }^{18}$, enjeu macrodiégétique de cette bande; c'est aussi ces courbures du point de vue, ces retours diagonaux en arrière, ces rebonds sur des voiles, des miroirs et des points de fuite. Le regard du lecteur est piégé, manipulé.

Sortie nue, l'image, on l'a vu(e), n'est toutefois pas la Vérité; espionne, elle génère plutôt le doute, se complaisant dans la complexité par enroulement sur soi, dans l'obliquité, dans la courbure d'un espace fourbe. "La vérité? Tu crois que ça existe?" [50:5]. Méfiance de l'image à l'endroit de la Vérité comme butée, à laquelle répond cette méfiance à l'endroit de l'image qu'avaient radicalisée à Byzance les iconoclastes et iconodules de la querelle des images. N'oubliez pas que la version $\mathrm{BD}$ et spionspiel de cette byzantine querelle se passe à Istanbulle...

18 Mari de Magda apprendra-t-on plus tard, quasi-mari en fait, comme dans le fameux Casablanca de Michael Curtiz, auquel ce ne sont pas seulement cette situation ou le visage de Magda qui renvoient. Casablanca comme case blanche? 